\title{
Citing Wikipedia
}

\author{
Don't do it-Wikipedians wouldn't
}

\author{
Lane Rasberry Wikipedian in residence \\ Consumer Reports, 101 Truman Avenue, Yonkers, NY 10703, USA
}

Wikipedia should not be cited as a source of information. It is a summary of primary and secondary sources, all of which should be referenced, so people wishing to cite information found in Wikipedia should follow its citation to the source from which it was derived and credit the original authors and work. Wikipedia was never intended for use as an independent authority because it is authored by anonymous online contributors. The Wikipedia community (anyone who contributes to or edits Wikipedia) seeks to back up every statement with citations and encourages all readers to verify what they read by going back to the original sources. Wikipedia helps readers to find sources to cite. It is not itself a citable source.

In a linked study (doi:10.1136/bmj.g1585), ${ }^{1}$ Bould and colleagues found more than 1400 health science articles that cited Wikipedia, with half of the citations occurring between 2010 and 2013. They categorized each citation by type and judged that just $4 \%$ of citations were appropriate-in other words, categorized as "citations about Wikipedia" or "Wikipedia used in methods." The headline message, which I believe to be true, is that Wikipedia is being cited unjustifiably with sufficient frequency to constitute a problem that merits a response from the academic community. The citing of Wikipedia not only shows a lack of recognition that Wikipedia is not a good source for citation but also signals a greater concern that some readers are failing to apply critical thinking to judge the quality of information they encounter.

Bould and colleagues conclude that health science journals should be cautious about publishing papers that cite Wikipedia. I also hope that this paper encourages researchers to spread the word, already accepted by the Wikipedia community, that Wikipedia is not to be cited in health science articles, or anywhere else. Perhaps researchers and academics cite Wikipedia not knowing that the content is compiled by anonymous contributors over the internet with no formal oversight. In which case, Wikipedians need to be clearer about the provenance of Wikipedia content. Alternatively, people may be citing Wikipedia while knowing that it is not authoritative, possibly because it has some special citation appeal not shared by other inappropriate sources. A final possibility is that all kinds of people, including academic authors, put faith in media without sufficiently considering the reliability of what they read or cite. If that is the case, then perhaps serious reflection is warranted in academia, research, and education generally. Bould and colleagues' findings indicate that we should all support each other in being vigilant to raise research standards.

Wikipedia's popularity tells me, as an enthusiastic contributor, that people are keen to learn and like having their information reliably sourced to credible research publications. Everyone who lives in an information environment influenced by Wikipedia should be aware that it is an online encyclopedia published within a platform that encourages its readers to make modifications to the publication as they read it. Anyone at any time can click the "edit" button at the top of any Wikipedia article and then change it as they like.

In traditional reference works, having personal authorship by experts is the main quality control strategy. Historically, publishers sought to avoid publishing anything written by unknown people with dubious credentials. Thanks to this precedent, some people may assume that information is dependable even though it is not offered in the same way by Wikipedia or by many other channels of new media. Wikipedia publishes through a structure of anonymous volunteer authors, so its strategy for maintaining integrity is to request exhaustive citing of sources for the information it presents. As a summary of published thought, Wikipedia does not publish anyone's original ideas or new interpretation. This design recruits readers who respect and defer to the original sources by strongly encouraging them to check the veracity of what they see by following citations. Also, as readers check a Wikipedia citation, they are automatically asked by the Wikipedia community either to endorse (by inaction) Wikipedia's text if they agree with it or to change the text if they find that it does not agree with the original source. This is how Wikipedia maintains its quality and integrity.

Wikipedia's pervasiveness, as documented in the linked paper, ${ }^{1}$ could be an opportunity for increased collaboration between the Wikipedia community and academia. Wikipedia is already the rare project that collects the leisure time of volunteers who enjoy discussing the relative merit of sources and championing the correct use of citations. Wikipedia's infrastructure is particularly good for teaching contributors the difference between primary, 
secondary, and tertiary sources and encouraging researchers to cite secondary sources such as review articles in preference to anything else. Anyone who wants to learn more about the Wikipedia project can post questions to Wikipedia's Reliable Sources Noticeboard (https://en.wikipedia.org/wiki/Wikipedia: Reliable_sources/Noticeboard), the Wikipedia Education Program (https://en.wikipedia.org/wiki/Wikipedia:Education_ program), which does outreach to universities, or the WikiProject Medicine forum (https://en.wikipedia.org/wiki/ Wikipedia:WikiProject_Medicine); or, better, they can add something to a health science article in Wikipedia and see the response. All are welcome.

Everyone should try to have research practices at least as good as those of the Wikipedians, and they would never cite Wikipedia.
Competing interests: I have read and understood the BMJ Group policy on declaration of interests and declare the following interests: I am employed by Consumer Reports with the title "Wikipedian in residence." In this role, I serve as liaison between the Wikimedia community and Consumer Reports as the two groups collaborate to achieve their mutual goals. I have never been employed by the Wikimedia Foundation but am a volunteer member of the Wikimedia community.

Provenance and peer review: Commissioned; not externally peer reviewed.

1 Bould MD, Hladkowicz ES, Pigford AE, Ufholz L, Postonogova T, Shin E, et al. References that anyone can edit: review of Wikipedia citations in peer reviewed health science literature. BMJ 2014;348:g1585.

Cite this as: BMJ 2014;348:g1819

(๑) BMJ Publishing Group Ltd 2014 